\title{
Research on Coal-Rock Fracture Image Edge Detection Based on Tikhonov Regularization and Fractional Order Differential Operator
}

\author{
Chunsheng Liu ${ }^{1}$ and Chunping Ren ${ }^{10}{ }^{1,2}$ \\ ${ }^{1}$ Heilongjiang University of Science and Technology, Harbin 150022, China \\ ${ }^{2}$ College of Mechanical and Electrical Engineering, Harbin Engineering University, Harbin 150001, China
}

Correspondence should be addressed to Chunping Ren; renchunpin@sina.com

Received 29 January 2019; Revised 13 March 2019; Accepted 11 April 2019; Published 2 May 2019

Academic Editor: Sos S. Agaian

Copyright (c) 2019 Chunsheng Liu and Chunping Ren. This is an open access article distributed under the Creative Commons Attribution License, which permits unrestricted use, distribution, and reproduction in any medium, provided the original work is properly cited.

\begin{abstract}
Aiming at the conventional image edge detection algorithm, the first-order differential edge detection method is easy to lose the image details and the second-order differential edge detection method is more sensitive to noise. To deal with the problem, the Tikhonov regularization method is adopted to reconstruct the input coal-rock infrared images, so as to reduce the noise interference, and then, the reconstructed image is transformed by gray level. Finally, we consider the frequency characteristics and long memory properties of fractional differential, the classical first-order Sobel and second-order Laplacian edge detection algorithms are extended to fractional order pattern, and a new pattern of fractional order differential image edge detection is constructed to realize the coal-rock fracture edge features identification. The results show that, compared with integer order differential, the error rate and omission rate of fractional order differential algorithm are smaller, the quality factor is larger, and the execution time and memory footprint are smaller. From the point of view of location criteria and location accuracy, the fractional order differential algorithm is better than the integer order. In addition, the proposed method is compared with Canny algorithm, B-spline wavelet transform, and multidirection fuzzy morphological edge detection method, can detect more coal-rock fracture infrared image edge details, and is more robust to noise.
\end{abstract}

\section{Introduction}

The coal-rock fracture detection is an effective means to help coal seam gas development, once coal-rock image fractures are accurately detected, which will play an important role in further exploiting coal seam gas $[1,2]$. In the final analysis, the coal-rock image fracture detection is edge detection, the method can be basically divided into two major categories, one of two methods is based on the firstorder derivative method, which detects the boundary by finding the maximum and minimum values of the image first-order derivative, and the boundary is usually positioned in the direction of the maximum gradient $[3,4]$. Another kind is the second-order derivative method; when the image first-order derivative is taken as the maximum, the second-order derivative is zero, so we can find the boundary by looking for the zero crossing point of the image second-order derivative [5]. These two methods are implemented by convoluting the specific template with the image to achieve the image boundary point detection, but there are differences in the detection effect. The first-order derivative method is easy to produce thicker edge, which results in the loss of part of the image information details. And the second-order derivative method has strong image detail detection ability, but it is very sensitive to noise [6].

Fractional order differential theory is a generalization of integral order differential theory, and it has been widely used in many research fields such as applied mathematics, medicine, and information science [7]. In recent years, image processing is a new research hotspot with fractional differential theory and has been successfully applied to solve many problems such as image processing, image 
enhancement, image denoising, and edge detection [8]. Bai and Feng [9] proposed an image denoising pattern based on fractional anisotropic diffusion equation and used the fractional Fourier transformation to solve the pattern, which effectively suppresses the "ladder effect" phenomenon produced by the traditional denoising method. Józwik [10] proposed a fractional order robust contour edge detector, which can selectively detect edges when the order of fractional differential is properly chosen. Chen et al. [11] constructed two different fractional edge detection operators using fractional differential instead of traditional firstorder differential. He et al. [12] proposed an edge detection operator based on compound derivative, which uses the combination of fractional differential and integral differential. Goddeke and Strzodka [13] introduced fractional calculus in the traditional Kalman filter, and a discrete fractional order Kalman filter algorithm is proposed in the linear space and nonlinear space to estimate the parameters and fractional order. In [14], fractional differential can be used to accurately detect the medical image edges and to effectively suppress noise, which will improve the speed and accuracy of medical diagnosis.

The above analysis shows that the edge detection method based on fractional order differential can not only effectively extract the image edge information but also preserve the image texture details, so fractional order differential edge detection is better than the integer order differential. Although the first-order Sobel and the second-order Laplacian operators are not ideal for edge detection, there is no denying that they still have a wide range of applications due to stronger universality and faster computing speed. Therefore, if the fractional order differential is introduced into the firstorder and the second-order differential operators, it will inherit the advantages of the first-order and second-order differential operators and improve the edge detection effect, so which can provide a new effective method to detect image edge.

In order to effectively and accurately detect the image edge, we were inspired by the fractional differential theory, the first-order Sobel edge detection operator and the secondorder Laplacian edge detection operator is extended to fractional order pattern, which is used to extract the edge feature of coal-rock fracture infrared image. The results show that, compared with integer order differential, fractional order differential can detect more image edge detail features and is more robust to noise.

\section{Image Reconstruction}

The image reconstruction model is uniformly described as follows [15-19]:

$$
A u=s,
$$

where $A$ represents a matrix of dimension $m \times n, u$ represents an $n \times 1$ dimensional vector denoting the gray level values, and $s$ represents an $m \times 1$ dimensional vector denoting the normalized capacitance values.

Image reconstruction is an ill-posed problem, and it is generally known that Tikhonov regularization is an efficient way to solve ill-posed problems. Its basic idea is to transform equation (1) into an optimization problem [20-24]:

$$
\min J(u)=\|A u-s\|^{2}+\lambda\|u\|^{2},
$$

where $\lambda$ is the regularization parameter and $\|\cdot\|$ denotes 1 norm. Then, minimizing equation (2) yields the solution:

$$
u=\left(A^{T} A+\lambda I\right)^{-1} A^{T} s
$$

In the MATLAB 7.0 environment, the Tikhonov regularization method is used to reconstruct the coal-rock infrared image.

Figure 1(a) shows the coal-rock fracture infrared image [25-27], Figure 1(b) represents the reconstructed image, and Figure 1(c) represents a gray-level transformation image. In Figure 1(a), we can see that the infrared image contains a lot of noise, and the reconstructed image noise is obviously reduced from Figure 1(b) using the Tikhonov regularization method. Therefore, our conclusion is that the Tikhonov regularization method can reduce the noise interference, which lays the foundation for further image edge detection. The edge detection mentioned below uses gray-level transformation images as input images.

\section{Image Edge Detection}

\subsection{Integer Order Edge Detection Operator}

3.1.1. First-Order Sobel Operator. Sobel edge detection method is a typical first-order gradient, it uses a pair of $3 \times 3$ mobile template in the image, and as a traditional method, the corresponding gradient based on each pixel as the center point can be calculated, finally produces the horizontal gradient map and vertical gradient map of image, respectively. For a continuous image $u(x, y)$, the gradient of position $(x, y)$ can be defined as the following form [28]:

$$
\nabla u(x, y)=\left[G_{x}, G_{y}\right]^{\mathrm{T}} \text {, }
$$

where $G_{x}, G_{y}$ represents the gradient along the $X$-axis and the $Y$-axis, respectively. In an image, if $\Delta x$ and $\Delta y$ are computed according to the number of pixels between two pixels, let $\Delta x=\Delta y=2$, and the differential form of the gradient component is represented as follows:

$$
\begin{aligned}
& G_{x}=\frac{1}{2}\left(\frac{\partial u(x+1, y-1)}{\partial x}+2 \frac{\partial u(x+1, y)}{\partial x}+\frac{\partial u(x+1, y+1)}{\partial x}\right), \\
& G_{y}=\frac{1}{2}\left(\frac{\partial u(x-1, y+1)}{\partial y}+2 \frac{\partial u(x, y+1)}{\partial y}+\frac{\partial u(x+1, y+1)}{\partial y}\right) .
\end{aligned}
$$

3.1.2. Second-Order Laplacian Operator. According to the calculus theory, the local maxima of the first-order derivative correspond to the zero crossing of the second-order derivative, so the image edge can be detected by the zero crossing point of the image second-order derivative. For a continuous image $u(x, y)$, the second-order derivative of position $(x, y)$ can be defined as the following form [29]: 


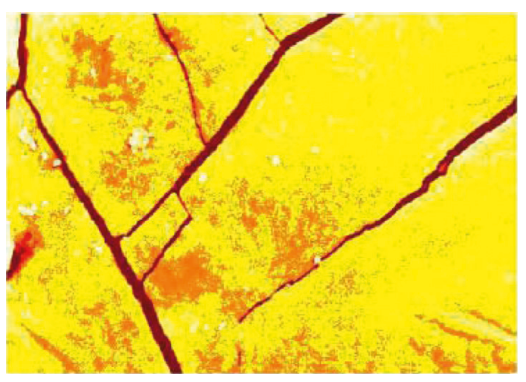

(a)

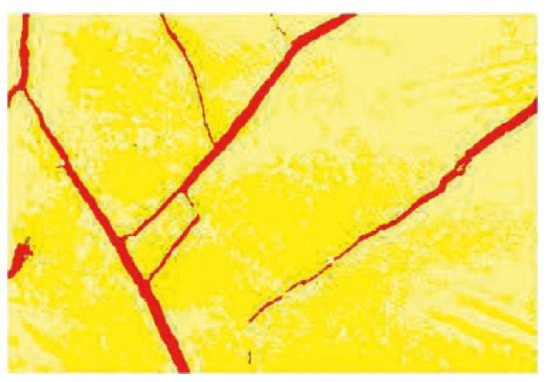

(b)

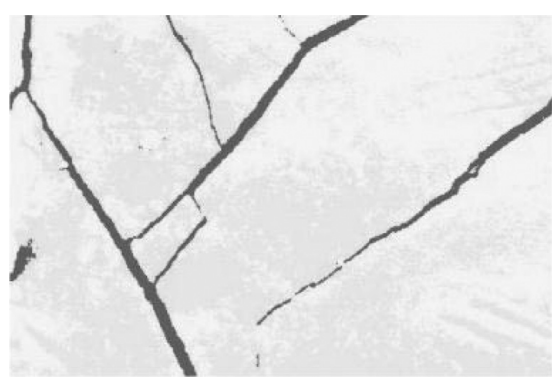

(c)

Figure 1: Coal-rock fracture image. (a) Infrared image. (b) Reconstructed image. (c) Gray-level transformation image.

$$
\nabla^{2} u(x, y)=\left[G_{x}^{2}, G_{y}^{2}\right]^{\mathrm{T}}
$$

where $G_{x}^{2}, G_{y}^{2}$ represents the second-order derivative along the $X$-axis and the $Y$-axis, respectively. In an image, if $\Delta x$ and $\Delta y$ are computed according to the number of pixels between two pixels, let $\Delta x=\Delta y=1$, and the differential form of the second-order derivative is represented as follows:

$$
G^{2}(u(x, y))=-\frac{\partial^{2} u(x+1, y)}{\partial x^{2}}-\frac{\partial^{2} u(x, y+1)}{\partial y^{2}} .
$$

3.2. Fractional Order Edge Detection Operator. Considering that the integer order differential can only deal with the image information in the eight neighborhoods, however, the fractional differential can deal with the image global information. In this paper, the traditional integer differential operator is extended to the fractional order pattern, which is beneficial to extract more image edge features.

3.2.1. Fractional Order Sobel Operator. By introducing differential order from first order to fractional order, a fractional Sobel operator is proposed, whose differential form is along the $X$-axis and the $Y$-axis [30-32]:

$$
\begin{aligned}
& G_{x}^{\alpha}=\frac{1}{2}\left(\frac{\partial^{\alpha} u(x+1, y-1)}{\partial x^{\alpha}}+2 \frac{\partial^{\alpha} u(x+1, y)}{\partial x^{\alpha}}+\frac{\partial^{\alpha} u(x+1, y+1)}{\partial x^{\alpha}}\right), \\
& G_{y}^{\alpha}=\frac{1}{2}\left(\frac{\partial^{\alpha} u(x-1, y+1)}{\partial y^{\alpha}}+2 \frac{\partial^{\alpha} u(x, y+1)}{\partial y^{\alpha}}+\frac{\partial^{\alpha} u(x+1, y+1)}{\partial y^{\alpha}}\right) .
\end{aligned}
$$

Fractional differential operators $G_{x}^{\alpha}$ and $G_{y}^{\alpha}$ are discretized by using fractional differential G-L. We assume the image size is $M \times N$, and then, fractional gradient at $(x, y)$ is represented as follows:

$$
\left(\nabla^{\alpha} u\right)_{i, j}=\left(\left(\Delta_{x}^{\alpha} u\right)_{i, j},\left(\Delta_{y}^{\alpha} u\right)_{i, j}\right), \quad 1 \leq i \leq M, 1 \leq j \leq N,
$$

where

$$
\begin{aligned}
\left(\Delta_{x}^{\alpha} u\right)_{i, j} & =\sum_{k=0}^{K-1} w_{j}^{(\alpha)} u_{i, j-k}, \\
\left(\Delta_{y}^{\alpha} u\right)_{i, j} & =\sum_{k=0}^{K-1} w_{j}^{(\alpha)} u_{i, j-k} .
\end{aligned}
$$

$K \geq 3$ is the integer constant, and $w_{j}^{(\alpha)}$ can be derived from the following recurrence formula:

$$
\begin{aligned}
& w_{0}^{(\alpha)}=1, \\
& w_{0}^{(\alpha)}=\left(1-\frac{\alpha+1}{j}\right) w_{j-1}^{(\alpha)}, \\
& \quad j=1,2, \ldots, K-1 .
\end{aligned}
$$

Then, the fractional gradient components along the $X$ axis and the $Y$-axis can be approximated as follows:

$$
\begin{aligned}
G_{x}^{\alpha}= & \frac{1}{2}\left[u(x-1, y+1)-\alpha u(x-1, y)+\frac{\alpha^{2}-\alpha}{2} u(x-1, y-1)\right. \\
& +\cdots+(-1)^{k} C_{k}^{\alpha} u(x-1, y+1-k)+2 u(x, y+1) \\
& -2 \alpha u(x, y)+\left(\alpha^{2}-\alpha\right) u(x, y+1)+\cdots \\
& +2 *(-1)^{k} C_{k}^{\alpha} u(x, y+1-k)+u(x+1, y+1) \\
& -\alpha u(x+1, y)+\frac{\alpha^{2}-\alpha}{2} u(x+1, y-1)+\cdots \\
& \left.+(-1)^{k} C_{k}^{\alpha} u(x+1, y+1-k)\right], \\
G_{y}^{\alpha}= & \frac{1}{2}\left[u(x+1, y-1)-\alpha u(x, y-1)+\frac{\alpha^{2}-\alpha}{2} u(x-1, y-1)\right. \\
& +\cdots+(-1)^{k} C_{k}^{\alpha} u(x+1-k, y-1)+2 u(x+1, y) \\
& -2 \alpha u(x, y)+\left(\alpha^{2}-\alpha\right) u(x-1, y)+\cdots \\
& +2 *(-1)^{k} C_{k}^{\alpha} u(x+1-k, y)+u(x+1, y+1) \\
& -\alpha u(x, y+1)+\frac{\alpha^{2}-\alpha}{2} u(x-1, y+1)+\cdots \\
& \left.+(-1)^{k} C_{k}^{\alpha} u(x+1-k, y+1)\right] .
\end{aligned}
$$


A fractional Sobel convolution template based on the above approximation is presented, as shown in Figure 2.

3.2.2. Fractional Order Laplacian Algorithm. The differential order is extended from the second order to the fractional order, a fractional Laplacian operator is proposed, whose differential form can be defined as follows [33, 34]:

$$
G^{\alpha}(u(x, y))=-\frac{\partial^{\alpha} u(x+1, y)}{\partial x^{\alpha}}-\frac{\partial^{\alpha} u(x, y+1)}{\partial y^{\alpha}} .
$$

Fractional order differential operator $G^{\alpha}$ is discretized using fractional order differential G-L. Then, fractional gradient is represented as follows:

$$
\begin{aligned}
G^{\alpha}(u)= & -\sum_{k=0}^{K-1} w_{j}^{(\alpha)} u(x+1-k, y)-\sum_{k=0}^{K-1} w_{j}^{(\alpha)} u(x, y+1-k) \\
= & -\left[u(x+1, y)-\alpha u(x, y)+\frac{\alpha^{2}-\alpha}{2} u(x-1, y)+\ldots\right. \\
& \left.+(-1)^{K-1} C_{K-1}^{\alpha} u(x+2-K, y)\right]-[u(x, y+1) \\
& -\alpha u(x, y)+\frac{\alpha^{2}-\alpha}{2} u(x, y-1)+\cdots \\
& \left.+(-1)^{K-1} C_{K-1}^{\alpha} u(x, y+2-K)\right]
\end{aligned}
$$

A fractional order Laplacian convolution template based on the above approximation is presented, as shown in Figure 3.

3.2.3. Threshold Selection. The image fractional order gradient amplitude is selected as the basis of judging the image edge point $[35,36]$ :

$$
\left|\nabla^{\alpha} u\right|=\left[\left(G_{x}^{\alpha}\right)^{2}+\left(G_{y}^{\alpha}\right)^{2}\right]^{1 / 2}
$$

This measure gives the maximum change rate of $u(x, y)$ in the unit distance along the $\nabla^{\alpha} u$ direction, where the threshold of the edge point is set based on the average fractional gradient, let

$$
T=\tau \sum^{M} \sum^{N} \nabla^{\alpha} \frac{u}{(M N)},
$$

where $T$ means the threshold, $\tau \geq 1$ is a preset parameter, $M \times N$ represents the image size, and pixels can be labeled as edge points when $\nabla^{\alpha} u>T$.

\section{Test Results and Analysis}

4.1. Selecting Fractional Order Differential Template Size. In order to investigate the fractional order differential template size (fractional differential expansion term $K$ ) influence on the results of image edge detection, we take the

\begin{tabular}{|c|c|c|c|c|}
\hline$\left((-1)^{k} C_{k}^{\alpha}\right) / 2$ & $\ldots$ & $\left(\alpha^{2}-\alpha\right) / 4$ & $-\alpha / 2$ & $1 / 2$ \\
& & & & \\
$(-1)^{k} C_{k}^{\alpha}$ & $\ldots$ & $\left(\alpha^{2}-\alpha\right) / 2$ & $-\alpha$ & 1 \\
& & & & $1 / 2$ \\
$\left((-1)^{k} C_{k}^{\alpha}\right) / 2$ & $\ldots$ & $\left(\alpha^{2}-\alpha\right) / 4$ & $-\alpha / 2$ & \\
& & & & \\
\hline
\end{tabular}

(a)

\begin{tabular}{|c|c|c|}
\hline$\left((-1)^{k} C_{k}^{\alpha}\right) / 2$ & $(-1)^{k} C_{k}^{\alpha}$ & $\left((-1)^{k} C_{k}^{\alpha}\right) / 2$ \\
\hline$\vdots$ & $\vdots$ & $\vdots$ \\
$\left(\alpha^{2}-\alpha\right) / 4$ & $\left(\alpha^{2}-\alpha\right) / 2$ & $\left(\alpha^{2}-\alpha\right) / 4$ \\
\hline$-\alpha / 2$ & $-\alpha$ & $-\alpha / 2$ \\
\hline $1 / 2$ & 1 & $1 / 2$ \\
\hline
\end{tabular}

(b)

FIGURE 2: Fractional order Sobel template. (a) $G_{x}^{\alpha}$. (b) $G_{y}^{\alpha}$.

\begin{tabular}{|c|c|c|c|c|}
\hline 0 & $\ldots$ & 0 & $(-1)^{k} C_{K-1}^{\alpha}$ & 0 \\
\hline$\vdots$ & $\vdots$ & $\vdots$ & $\vdots$ & $\vdots$ \\
\hline 0 & $\ldots$ & 0 & $\left(\alpha-\alpha^{2}\right) / 2$ & 0 \\
\hline$(-1)^{k} C_{K-1}^{\alpha}$ & $\ldots$ & $\left(\alpha-\alpha^{2}\right) / 2$ & $2 \alpha$ & -1 \\
\hline 0 & $\ldots$ & & & \\
\hline & & & & \\
\hline
\end{tabular}

Figure 3: Fractional order Laplacian template.

gray-level transformation coal-rock fracture image (size is $258 \times 258)$ as the test image. Figures 4 and 5 show the edge detection results of fractional order Sobel operator and fractional order Laplacian operator under the action of $3 \times 3$, $4 \times 4,5 \times 5,6 \times 6,7 \times 7$, and $8 \times 8$ templates, respectively. Parameters are as follows: $\tau=1.5$, using fractional order Sobel operator $\alpha=0.6$, and using fractional order Laplacian operator $\alpha=1.6$. 


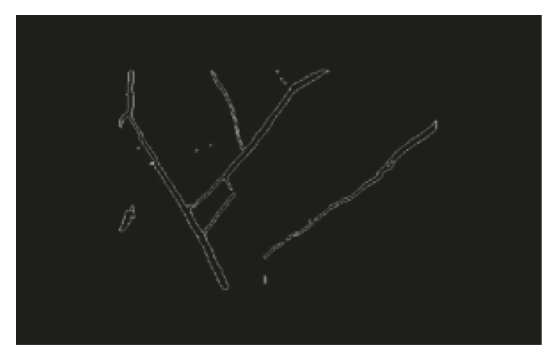

(a)

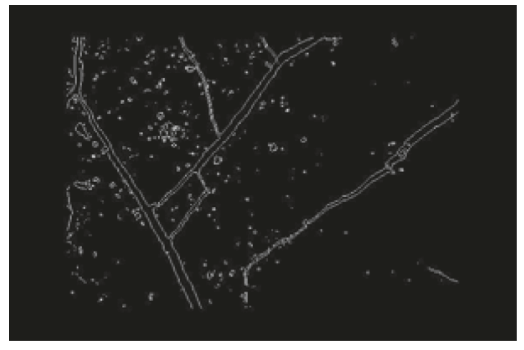

(d)

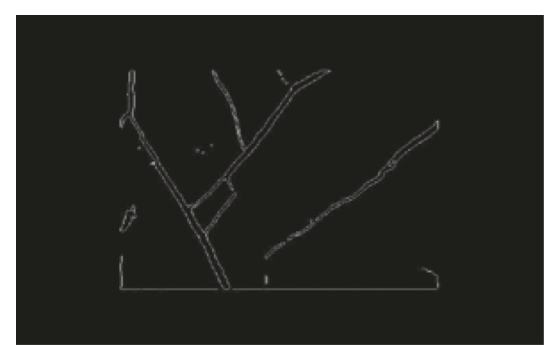

(b)

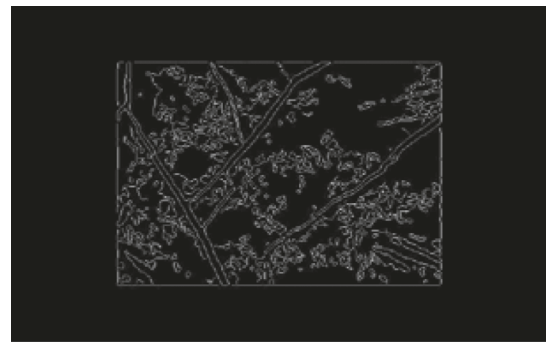

(e)

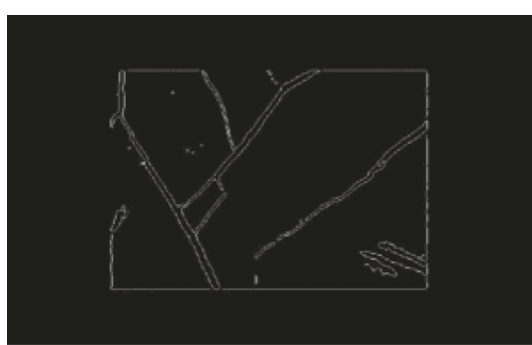

(c)

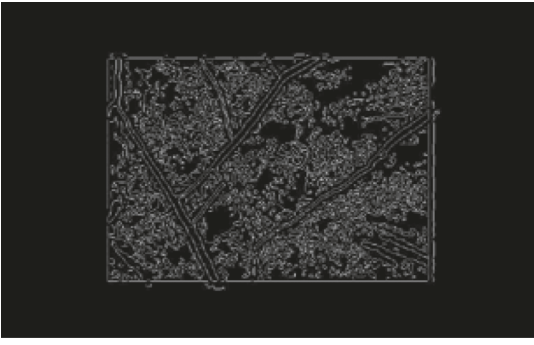

(f)

Figure 4: Edge extraction results of fractional order Sobel operator. (a) $3 \times 3$. (b) $4 \times 4$. (c) $5 \times 5$. (d) $6 \times 6$. (e) $7 \times 7$. (f) $8 \times 8$.

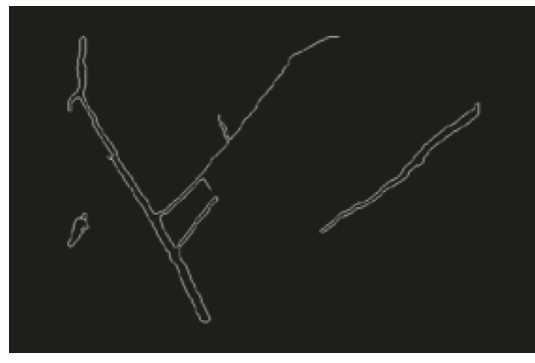

(a)

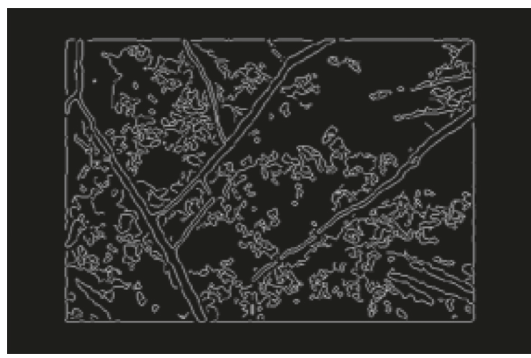

(d)

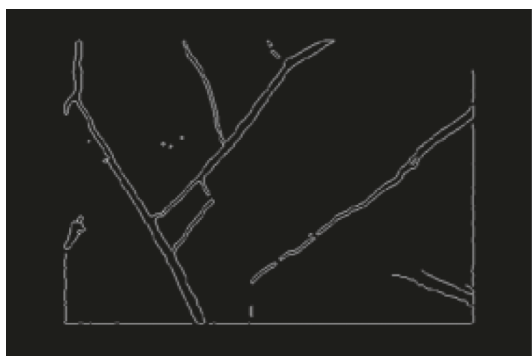

(b)

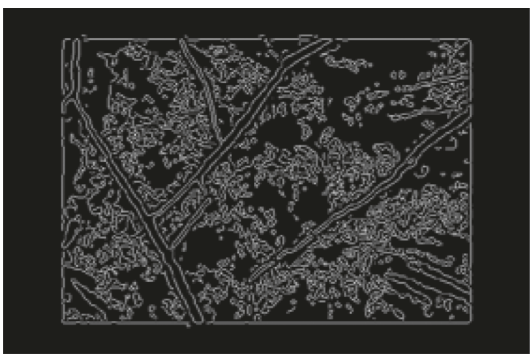

(e)

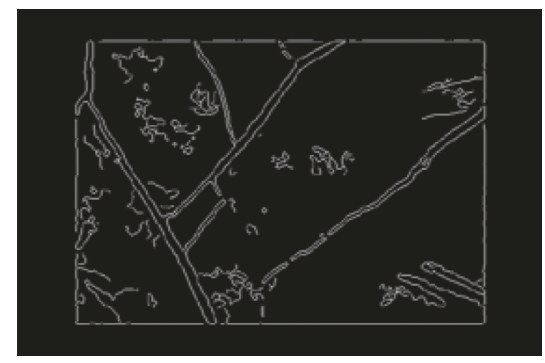

(c)

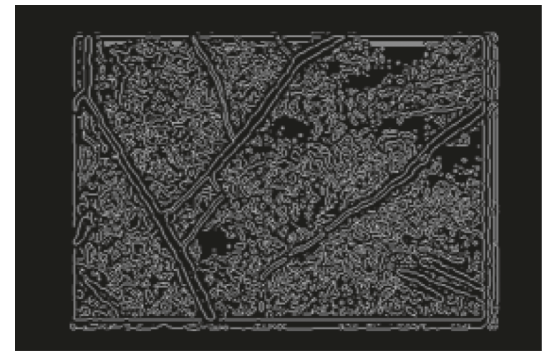

(f)

Figure 5: Edge extraction results of fractional order Laplacian operator. (a) $3 \times 3$. (b) $4 \times 4$. (c) $5 \times 5$. (d) $6 \times 6$. (e) $7 \times 7$. (f) $8 \times 8$.

In Figures 4 and 5, we can see that the edges of the image can be identified, but with the increasing of the template size, the edge of the recognition is confused, and the useless edge information is doped, which leads to the fact that the useful real edges are hidden. In other words, the fractional order differential template size is too large, there will be a lot of noise in the edge detection pattern when dealing with the noisy image, and the edge recognition will also consume a lot of computing time. Therefore, a small template size can effectively identify the true edges of an image, and it is appropriate to select the $3 \times 3$ template.
4.2. Fractional Image Edge Detection Results. Taking into account the frequency characteristics of the fractional differential and the global range of the domain, the classical first-order Sobel operator is extended to the fractional order pattern. Then, the performance and superiority of fractional Sobel operator edge detection are tested and analyzed by numerical experiments. The experimental parameters are set as follows: $K=3$ and differential order $\alpha \in(0,1)$. Firstly, the coal-rock fracture image in Figure 4(a) is chosen as the test image; let $\tau=3$. In Figure 6, the results of fractional Sobel operators under several typical fractional orders are given. 


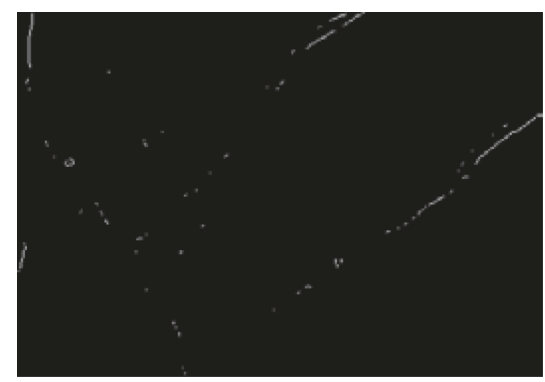

(a)

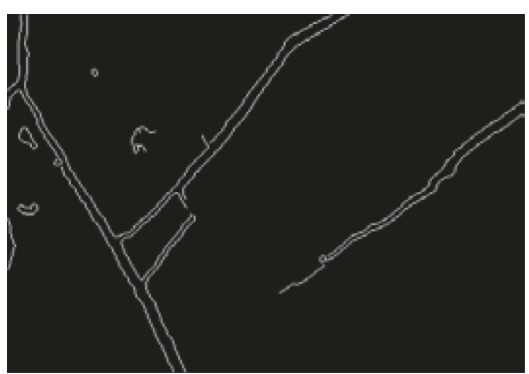

(d)

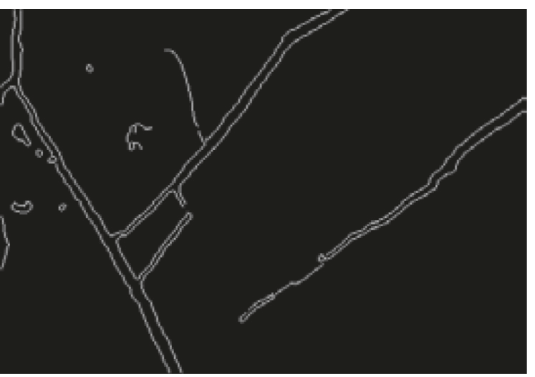

(g)

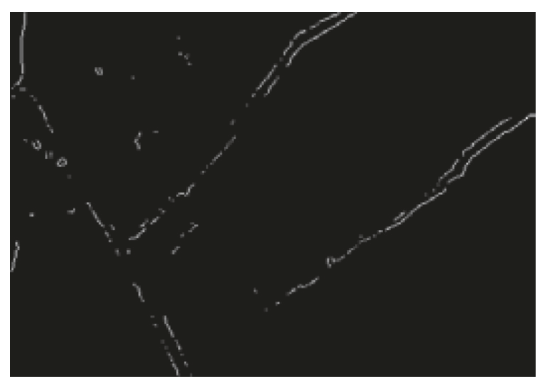

(b)

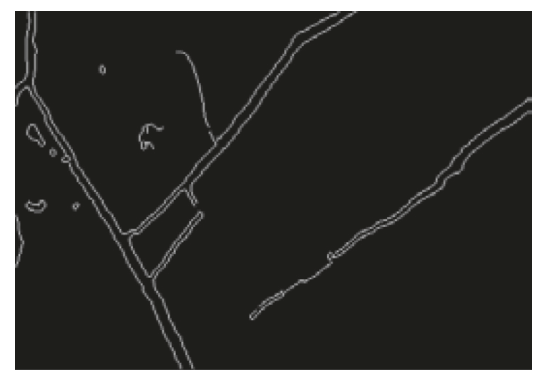

(e)

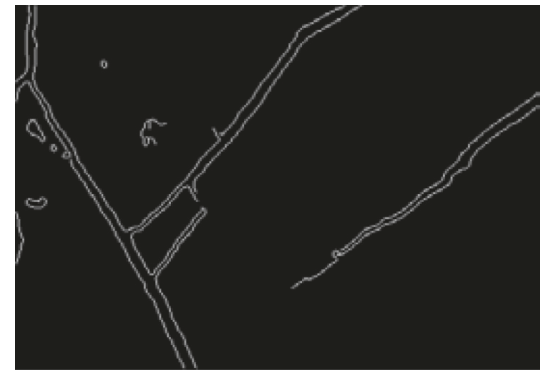

(h)

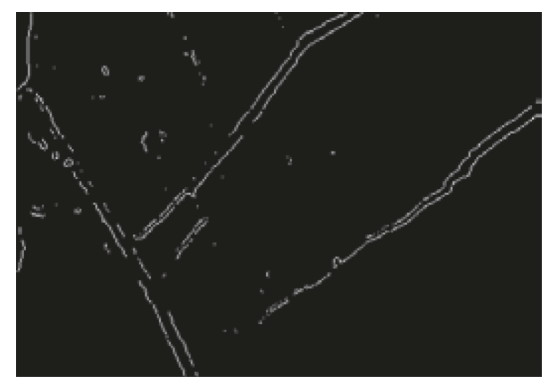

(c)

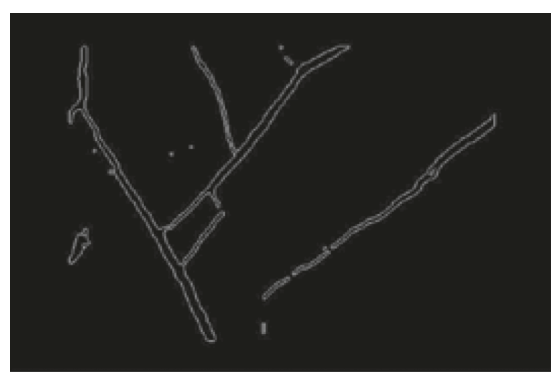

(f)

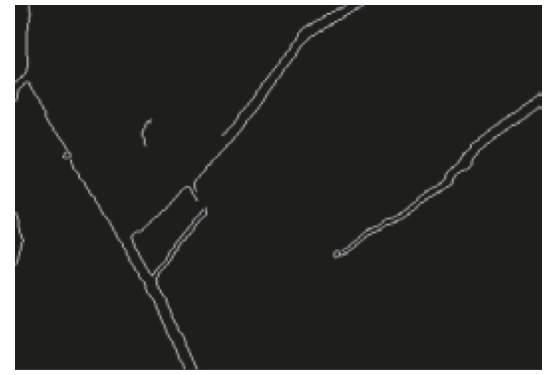

(i)

Figure 6: Comparison of edge extraction results under different orders Sobel on a coal-rock image. (a) $\alpha=0.1$. (b) $\alpha=0.2$. (c) $\alpha=0.3$. (d) $\alpha=0.4$. (e) $\alpha=0.5$. (f) $\alpha=0.6$. (g) $\alpha=0.7$. (h) $\alpha=0.8$. (i) $\alpha=0.9$.

Experimental results show that, compared with the classical first-order Sobel operator, the fractional Sobel operator is more robust to noise and can effectively suppress the interference of irrelevant details. For fractional Sobel operators, with the increasing of the fractional order, the edge detail extraction capability of the image is enhanced, but the sensitivity to noise is also increased, and a large number of noise components are left in the image. This result agrees with the frequency characteristic of the fractional differential.

The performance and superiority of fractional Laplacian operator edge detection are tested and analyzed. The experimental parameters are set as follows: $K=3$ and differential order $\alpha \in(1.0,2.0)$. The results of fractional Sobel operators under several typical fractional orders are shown in Figure 7.

4.3. Quantitative Analysis. In order to quantitatively analyze the effect of fractional order edge detection, we give the following evaluation criteria including the edge localization criterion and location accuracy, the quality factor, execution time, and memory footprint.
Fractional differential algorithm performance is quantitatively measured by the edge localization criterion and location accuracy, and the noise effect is investigated. The edge localization criterion can detect a given object edge according to the edge detection algorithm, and location errors include real edge error detection and nonedge error detection, that is, missed detection and false detection.

Tables 1 and 2 show the results of the missed detection rate and false detection rate under different detection operators. In Table 1 , the missed detection rate and false detection rate of $0.1,0.2,0.3,0.4$, and 0.5 are larger than that of 0.6. However, the missed detection rate and false detection rate of 0.6 are smaller than that of $0.7,0.8$, and 0.9 . Hence, we find from Table 1 that the fractional order $\alpha=$ 0.6 has the lowest missed detection rate and false detection rate.

And it can be shown that the missed detection rate and false detection rate from Table 2 under fractional order Laplacian detection algorithm and the missed detection rate and false detection rate of 1.1, 1.2, 1.3, 1.4, and 1.5 are larger than that of 1.6. However, the missed detection rate and false detection rate of 1.6 are smaller than that of 1.7, 1.8, and 1.9. 


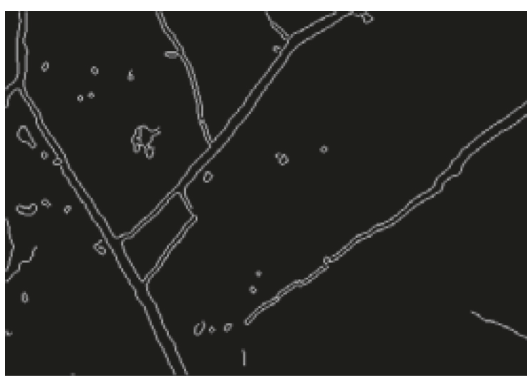

(a)

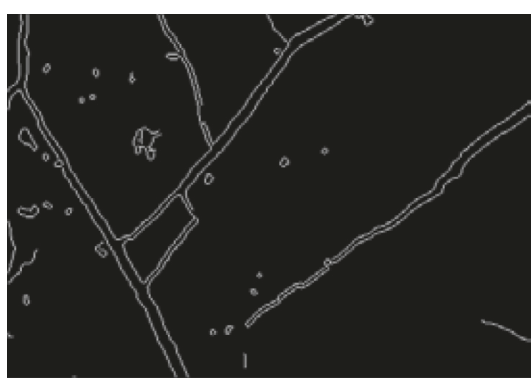

(d)

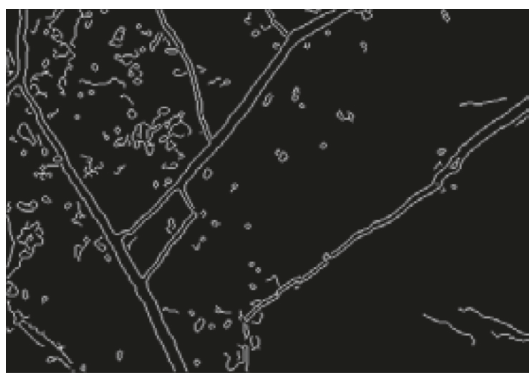

(g)

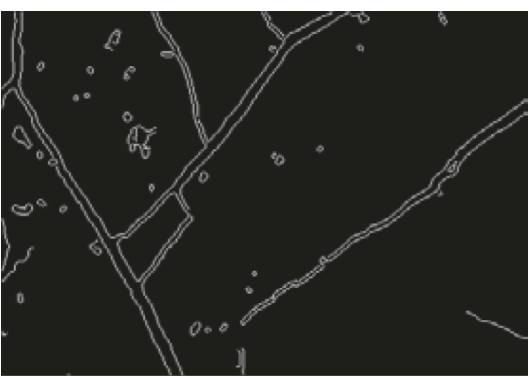

(b)

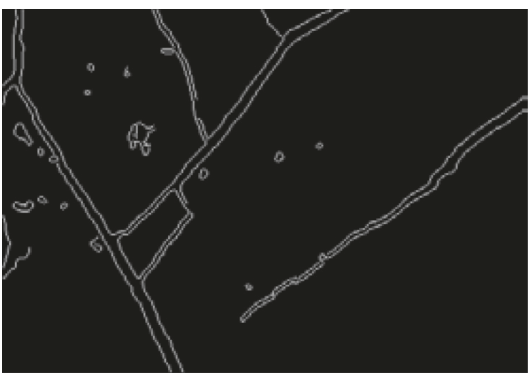

(e)

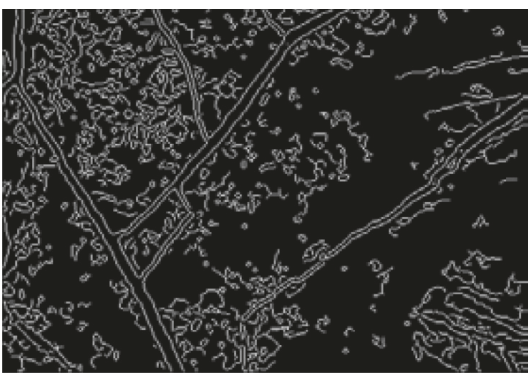

(h)

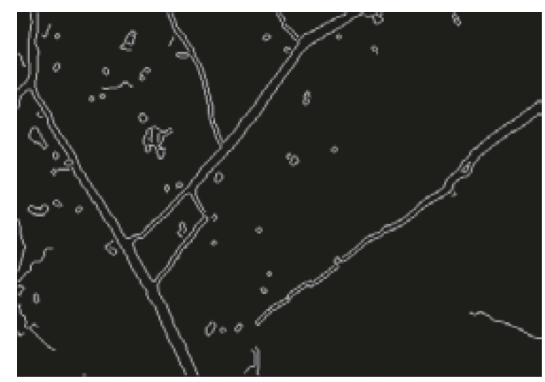

(c)

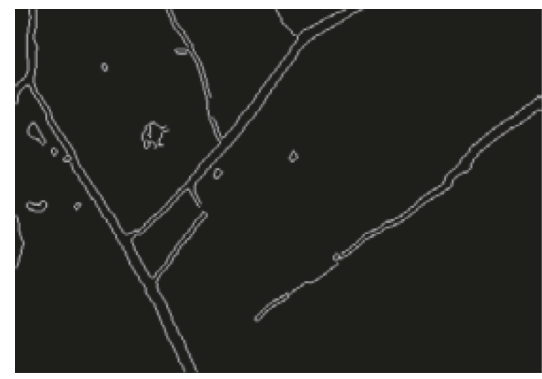

(f)

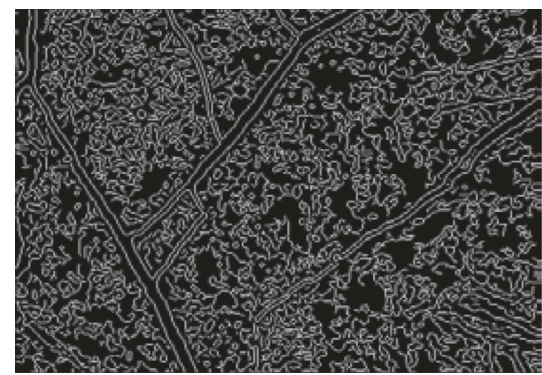

(i)

Figure 7: Comparison of edge extraction results under different orders Laplacian on a coal-rock image. (a) $\alpha=0.1$. (b) $\alpha=0.2$. (c) $\alpha=0.3$. (d) $\alpha=0.4$. (e) $\alpha=0.5$. (f) $\alpha=0.6$. (g) $\alpha=0.7$. (h) $\alpha=0.8$. (i) $\alpha=0.9$.

TABLE 1: Localization errors of fractional Sobel detection.

\begin{tabular}{lcc}
\hline Order & Missed detection rate & False detection rate \\
\hline 0.1 & 0.3574 & 0.6481 \\
0.2 & 0.3315 & 0.6328 \\
0.3 & 0.3104 & 0.6266 \\
0.4 & 0.3098 & 0.6099 \\
0.5 & 0.3032 & 0.6057 \\
0.6 & 0.3001 & 0.6009 \\
0.7 & 0.3256 & 0.6233 \\
0.8 & 0.3463 & 0.6446 \\
0.9 & 0.3519 & 0.6499 \\
\hline
\end{tabular}

Hence, we find from Table 2 that the fractional order $\alpha=1.6$ has the lowest missed detection rate and false detection rate.

Taking into account the above analysis, we can draw a conclusion that the optimal fractional orders $(\alpha=0.6$ and $\alpha=1.6$ ) using the above methods make them more successful to identify the edge of coal-rock fractures well.

In order to compare the performance of the two operators from the point of view of localization accuracy, the quality factor for edge detection is described as follows:
TABLE 2: Localization errors of fractional Laplacian detection.

\begin{tabular}{lcc}
\hline Order & Missed detection rate & False detection rate \\
\hline 1.1 & 0.4216 & 0.4785 \\
1.2 & 0.4183 & 0.4611 \\
1.3 & 0.4112 & 0.4589 \\
1.4 & 0.4085 & 0.4510 \\
1.5 & 0.4002 & 0.4497 \\
1.6 & 0.3991 & 0.4399 \\
1.7 & 0.4741 & 0.4871 \\
1.8 & 0.4824 & 0.5024 \\
1.9 & 0.5019 & 0.5112 \\
\hline
\end{tabular}

$$
Q=\frac{1}{\max \left(n_{\mathrm{a}}, n_{\mathrm{d}}\right)} \sum_{i=1}^{n_{\mathrm{d}}} \frac{1}{1+f d^{2}(i)},
$$

where $n_{\mathrm{a}}$ and $n_{\mathrm{d}}$ represent the actual edge number and the detected edge number, respectively, $d$ represents the distance between the real edge and the detected edge, $f$ is the constant factor, and $f=0.05$. If $Q$ is relatively larger, it means that localization accuracy of the edge points is higher; therefore, edges can be clearly detected. 
The quality factor is calculated according to equation (17), and the result is shown in Figure 8. In Figure 8(a), the quality factors of $0.1,0.2,0.3,0.4$, and 0.5 are smaller than that of 0.6 . However, the quality factor of 0.6 is larger than that of $0.7,0.8$, and 0.9. Hence, we find from Figure 8(a) that the fractional order $\alpha=0.6$ has the largest quality factor.

And it can be shown that the quality factor from Figure 8(b) under fractional order Laplacian detection algorithm and the quality factor of 1.1, 1.2, 1.3, 1.4, and 1.5 are smaller than that of 1.6. However, the quality factor of 1.6 is larger than that of 1.7, 1.8, and 1.9. Hence, we find from Figure 8 (b) that the fractional order $\alpha=1.6$ has the largest quality factor.

Taking into account the above analysis, we can draw a conclusion that the optimal fractional orders $(\alpha=0.6$ and $\alpha=1.6$ ) using the above methods make them more successful to identify the edge of coal-rock fractures well from the point of view of location accuracy.

Execution time and memory footprint not only reflect the algorithm computational complexity and execution efficiency but also reflect the algorithm performance. All codes were written in MATLAB 7.0 and run on a HP with 2.0 GB RAM and Windows 7 operating system. The test results of execution time and memory footprint are shown in Tables 3 and 4.

In Table 3, execution time and memory footprint of 0.1 , $0.2,0.3,0.4$, and 0.5 are larger than that of 0.6 . However, the quality factor of 0.6 is smaller than that of $0.7,0.8$, and 0.9 . Hence, we find from Table 3 that the fractional order $\alpha=0.6$ has the smallest execution time and memory footprint.

And it can be shown that the execution time and memory footprint from Table 4 under fractional order Laplacian detection algorithm and the execution time and memory footprint of $1.1,1.2,1.3,1.4$, and 1.5 are smaller than that of 1.6. However, the execution time and memory footprint of 1.6 are larger than that of 1.7, 1.8, and 1.9. Hence, we find from Table 4 that the fractional order $\alpha=1.6$ has the smallest execution time and memory footprint.

Taking into account the above analysis, we can draw a conclusion that the optimal fractional orders $(\alpha=0.6$ and $\alpha=1.6$ ) using the above methods make them more successful to identify the edge of coal-rock fractures well.

The fractional order edge detection method in this paper is compared with the classical first-order Sobel operator and second-order Laplacian edge detection operator, and the detection results of those methods are shown in Figures 9(a)-9(d), respectively.

Figure 9(a) explains that the detection result is gained using the classical first-order Sobel operator method at $\alpha=1.0$; Figure 9(b) explains that the detection result is given by the fractional order Sobel operator method at $\alpha^{*}=0.6$; Figure 9(c) shows that the detection result is gained using the second-order Laplacian edge detection operator at $\alpha=2.0$; and the detection result at $\alpha^{*}=1.6$ is shown in Figure 9(d) using the fractional order Laplacian edge detection operator in the paper.

We can find from Figures 9(a)-9(d) that the four methods can all detect the image edge; however, fractional order Sobel operator method and the fractional order Laplacian edge detection operator in the paper can successfully and effectively detect the detailed information of

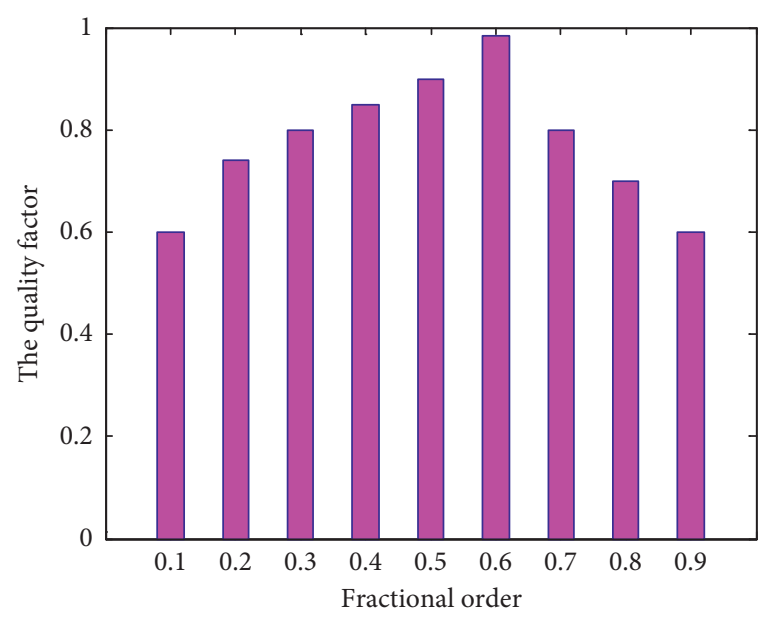

(a)

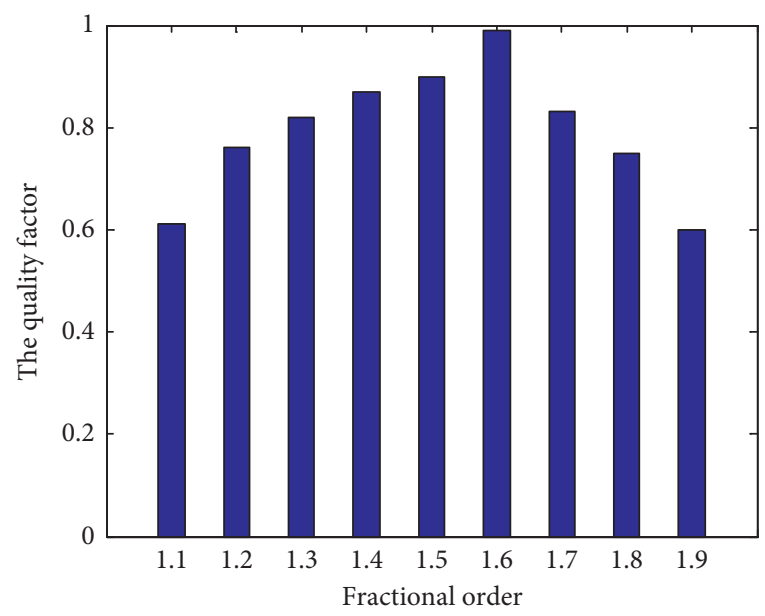

(b)

Figure 8: Quality factor. (a) Fractional order Sobel detection algorithm. (b) Fractional order Laplacian detection algorithm.

TABLE 3: Execution time and memory footprint of fractional Sobel detection.

\begin{tabular}{lcc}
\hline Order & Execution time $(\mathrm{s})$ & Memory footprint $(\mathrm{MB})$ \\
\hline 0.1 & 0.1766 & 33.92 \\
0.2 & 0.1665 & 33.01 \\
0.3 & 0.1559 & 32.65 \\
0.4 & 0.1407 & 31.27 \\
0.5 & 0.1297 & 30.89 \\
0.6 & 0.1288 & 29.76 \\
0.7 & 0.1441 & 31.77 \\
0.8 & 0.1626 & 32.18 \\
0.9 & 0.1936 & 34.48 \\
\hline
\end{tabular}

the image edge in comparison with the classical first-order Sobel operator and second-order Laplacian edge detection operator method.

In order to quantitatively analyze the effect of fractional order and integer order edge detection, we still give the following evaluation criteria including the localization errors, execution time and memory footprint, and the quality factor, which are shown in Tables 5-7. 
TABLE 4: Execution time and memory footprint of fractional Laplacian detection.

\begin{tabular}{lcc}
\hline Order & Execution time (s) & Memory footprint (MB) \\
\hline 1.1 & 0.2098 & 37.12 \\
1.2 & 0.2056 & 36.95 \\
1.3 & 0.2011 & 36.07 \\
1.4 & 0.1992 & 35.19 \\
1.5 & 0.1881 & 34.89 \\
1.6 & 0.1772 & 33.91 \\
1.7 & 0.1956 & 35.22 \\
1.8 & 0.2077 & 36.18 \\
1.9 & 0.2103 & 38.35 \\
\hline
\end{tabular}

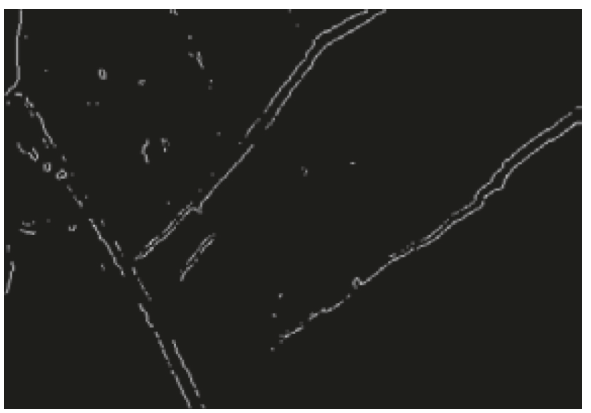

(a)

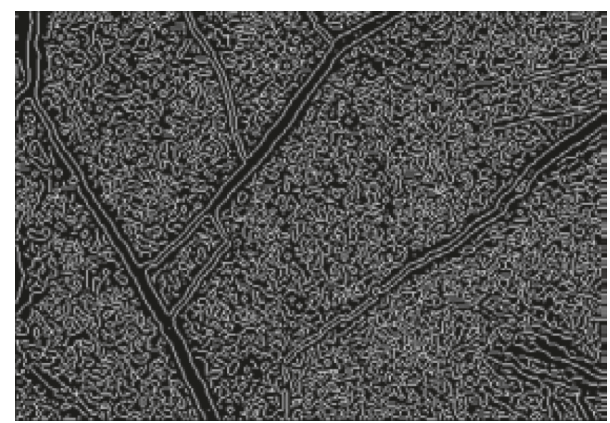

(c)

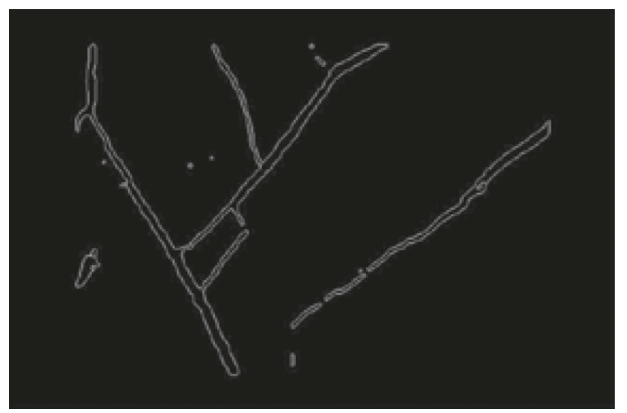

(b)

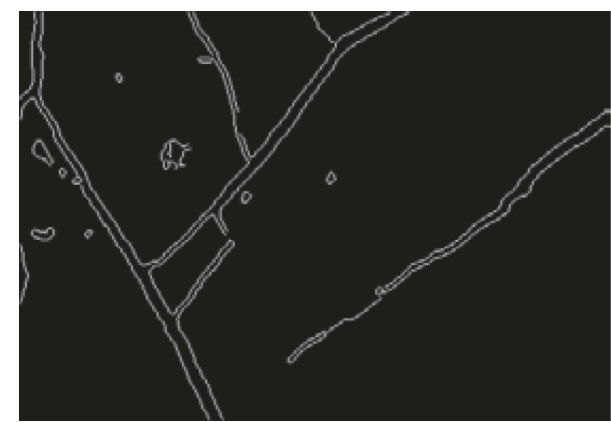

(d)

FIgURe 9: The comparison of integer order and fractional order methods. (a) $\alpha=1.0$. (b) $\alpha=0.6$. (c) $\alpha=2.0$. (d) $\alpha=1.6$.

TABLE 5: Localization errors of integer order and fractional order methods.

\begin{tabular}{|c|c|c|c|c|}
\hline $\begin{array}{l}\text { Evaluation } \\
\text { metrics }\end{array}$ & $\begin{array}{c}\text { The first-order Sobel } \\
\text { operator }(\alpha=10)\end{array}$ & $\begin{array}{c}\text { The fractional order Sobel } \\
\text { operator }\left(\alpha^{*}=0.6\right)\end{array}$ & $\begin{array}{l}\text { The second-order Laplacian } \\
\text { operator }(\alpha=2.0)\end{array}$ & $\begin{array}{c}\text { The fractional order Laplacian } \\
\text { operator }\left(\alpha^{*}=1.6\right)\end{array}$ \\
\hline $\begin{array}{l}\text { Missed } \\
\text { detection rate }\end{array}$ & 0.3677 & 0.3001 & 0.5136 & 0.3991 \\
\hline $\begin{array}{l}\text { False } \\
\text { detection rate }\end{array}$ & 0.6541 & 0.6009 & 0.5228 & 0.4399 \\
\hline
\end{tabular}

It is clearly seen from Tables 5 and 6 that the location errors (missed detection rate and false detection rate) and execution time and memory footprint of fractional order are less than that of the integer order detection algorithm. In Table 7 , we can see that the quality factor of fractional order is large than that of the integer order detection algorithm. Therefore, we conclude that the fractional order detection algorithm is better than the integer order detection algorithm which is more conducive to detect image edge.

\section{Comparison of Different Edge Detection Methods}

In order to compare the proposed method with other edge detection methods, this paper presents three recent edge detection methods, including Canny algorithm, B-spline wavelet transform, and multidirection fuzzy morphological edge detection method; the following are the steps of three edge detection algorithms: 
TABLE 6: Execution time and memory footprint of integer order and fractional order methods.

\begin{tabular}{lcccc}
\hline Evaluation metrics & $\begin{array}{c}\text { The first-order Sobel } \\
\text { operator }(\alpha=1.0)\end{array}$ & $\begin{array}{c}\text { The fractional order } \\
\text { Sobel operator }\left(\alpha^{*}=0.6\right)\end{array}$ & $\begin{array}{c}\text { The second-order } \\
\text { Laplacian operator }(\alpha=2.0)\end{array}$ & $\begin{array}{c}\text { The fractional order } \\
\text { Laplacian operator }\left(\alpha^{*}=1.6\right)\end{array}$ \\
\hline Execution time (s) & 0.2059 & 0.1288 & 0.2577 & 0.1772 \\
Memory footprint (MB) & 35.29 & 29.76 & 40.02 & 33.91 \\
\hline
\end{tabular}

TABLE 7: The quality factor of integer order and fractional order methods.

\begin{tabular}{lcccc}
\hline $\begin{array}{l}\text { Evaluation } \\
\text { metrics }\end{array}$ & $\begin{array}{c}\text { The first-order Sobel } \\
\text { operator }(\alpha=1.0)\end{array}$ & $\begin{array}{c}\text { The fractional order Sobel } \\
\text { operator }\left(\alpha^{*}=0.6\right)\end{array}$ & $\begin{array}{c}\text { The second-order Laplacian } \\
\text { operator }(\alpha=2.0)\end{array}$ & $\begin{array}{c}\text { The fractional order Laplacian } \\
\text { operator }\left(\alpha^{*}=1.6\right)\end{array}$ \\
$\begin{array}{l}\text { The quality } \\
\text { factor }\end{array}$ & 0.59 & 0.99 & 0.62 & 0.98 \\
\hline
\end{tabular}

The steps of Canny operator edge detection are as follows:

Step 1. The original image is smoothed or convoluted by using two-dimensional Gauss filtering function, which can reduce noise.

Step 2. Calculating the partial derivative $(G(x), G(y))$ of gray image, the gradient $\|G\|=\sqrt{G_{x}^{2}+G_{y}^{2}}$, and the direction $\theta=\arctan \left(G_{x} / G_{y}\right)$ of the gradient.

Step 3. We can get the direction of the edge by step 2 . The direction of edge gradient is divided into four directions: horizontal direction, vertical direction, 45 degree direction, and 135 degree direction.

Step 4. Nonmaximum suppression of image: if the gray value difference between a certain pixel and two adjacent pixels is not very large, then it is determined that the pixel is not an edge.

Step 5. Two thresholds are obtained by using cumulative histogram, including high threshold $\mathrm{T} 1$ and low threshold T2. If the value is greater than the high threshold $\mathrm{T} 1$, the edge is determined; if the value is smaller than the low threshold T2, the edge is not determined; and if the value is between the high threshold $\mathrm{T} 1$ and the low threshold T2, the two adjacent pixels are considered. If the two adjacent pixels are larger than the high threshold T1, the edge is determined; otherwise, the point is not considered as the edge point.

The steps of B-spline wavelet transform edge detection are as follows:

Step 1. The original image is transformed by wavelet transform, and the module image and phase image cluster are obtained.

Step 2. Find out the local modulus maximum point in the modulus image cluster and get the edge image to be selected.

Step 3. The mean value of modulus maxima is obtained by dividing blocks and weighted summation, and the threshold of each block is obtained.

Step 4. Remove false edges. Judging whether the selected edge point is the real edge point according to the threshold value obtained by each block, retaining the point whose modulus is greater than the threshold value, removing the false edge point which is less than the threshold value, and obtaining the real edge image.

The steps of multidirectional fuzzy morphological edge detection are as follows:

Step 1. Edge $E_{i}=\mu_{\left(F \cdot B_{i}\right) \otimes B_{i}}-\mu_{\left(F \cdot B_{i}\right) \otimes B_{i}}$ in all directions was detected.

Step 2. According to the detection edge result $E_{i}$, the final result $E=\sum_{i=1}^{4} \omega_{i} E_{i}$ is calculated by using composite weighting method.

Step 3. The detection results are binarized by using the Otsu method.

The proposed method is compared with other edge detection methods, the detection results of those methods are shown in Figures 10(a)-10(d), respectively.

Figure 10(a) explains that the detection result is gained using the classical Canny edge detection method; Figure 10(b) explains that the detection result is given by the B-spline wavelet transform edge detection method; Figure 10(c) shows that the detection result is gained using the multidirection fuzzy morphological edge detection method; and the detection result is shown in Figure 10(d) using the proposed edge detection operator in the paper.

We can find from Figures 10(a)-10(d) that the four methods can all detect the image edge; however, the proposed edge detection method in the paper can successfully and effectively detect the detailed information of the image edge in comparison with the classical Canny edge detection method, B-spline wavelet transform, and multidirection fuzzy morphological method.

In order to quantitatively analyze the effect of different edge detection methods, we still give the following evaluation criteria including the localization errors, execution time and memory footprint, and the quality factor, which are shown in Tables 8-10.

It is clearly seen from Tables 8 and 9 that the location errors (missed detection rate and false detection rate) and execution time and memory footprint of the proposed edge detection method in the paper are less than that of other methods. In Table 10, we can see that the quality factor of the 


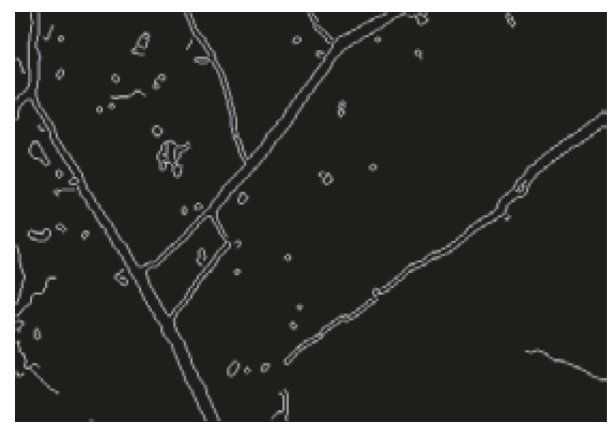

(a)

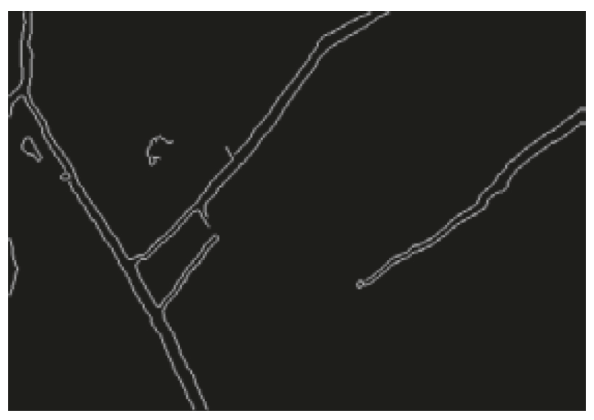

(c)

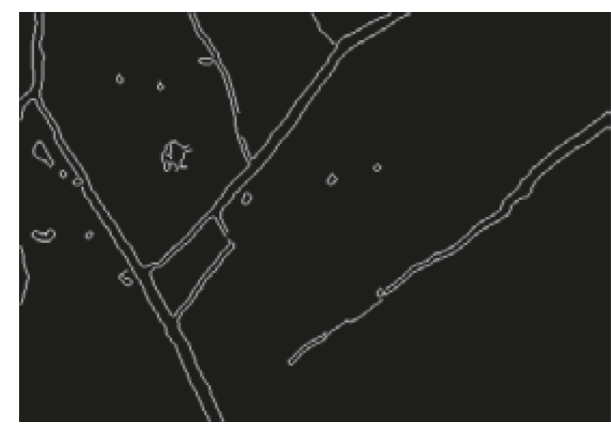

(b)

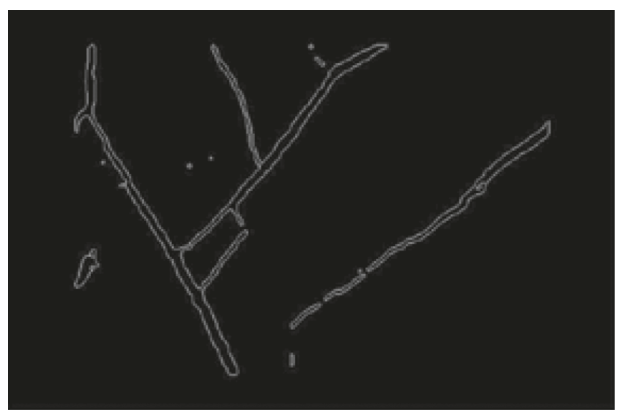

(d)

Figure 10: The comparison of different edge detection methods. (a) Canny method. (b) B-spline wavelet transform. (c) Multidirection fuzzy morphological method. (d) Present method.

TABLE 8: Localization errors under different edge detection methods.

\begin{tabular}{lcccc}
\hline Evaluation metrics & Canny & B-spline wavelet transform & Multidirection fuzzy morphological & Present \\
\hline Missed detection rate & 0.4836 & 0.4012 & 0.3378 & 0.3001 \\
False detection rate & 0.7977 & 0.7152 & 0.6815 & 0.6009 \\
\hline
\end{tabular}

TABLE 9: Execution time and memory footprint under different edge detection methods.

\begin{tabular}{lcccc}
\hline Evaluation metrics & Canny & B-spline wavelet transform & Multidirection fuzzy morphological & Present \\
\hline Execution time (s) & 0.2133 & 0.1906 & 0.1577 & 0.1288 \\
Memory footprint (MB) & 40.65 & 38.33 & 32.14 & 29.76 \\
\hline
\end{tabular}

TABLE 10: The quality factor under different edge detection methods.

\begin{tabular}{lcccc}
\hline Evaluation metrics & Canny & B-spline wavelet transform & Multidirection fuzzy morphological & Present \\
\hline The quality factor & 0.67 & 0.79 & 0.90 & 0.99 \\
\hline
\end{tabular}

proposed edge detection method in the paper is large than that of other methods. Therefore, we conclude that the proposed edge detection method in the paper is better than Canny method, B-spline wavelet transform, and multidirection fuzzy morphological edge detection method which is more conducive to detect image edge.

\section{Conclusions}

This paper presents a new pattern of fractional order differential image edge detection method to detect the edge of coal- rock fracture. Considering the frequency characteristics and long memory properties of fractional differential, integer order differential edge detection methods (first-order
Sobel and second-order Laplacian) are extended to the fractional order pattern. The test results show that, compared with integer order differential, the error rate and omission rate of fractional order differential algorithm are smaller, the quality factor is larger, and the execution time and memory footprint are smaller. From the point of view of location criteria and location accuracy, the fractional order differential algorithm is better than the integer order. In addition, the proposed method is compared with Canny algorithm, B-spline wavelet transform, and multidirection fuzzy morphological edge detection method; the proposed method in this paper based on Tikhonov regularization and fractional order differential operator is an effective method for image edge detection. 


\section{Data Availability}

No data were used to support this study.

\section{Conflicts of Interest}

The authors declare that there are no conflicts of interest.

\section{Acknowledgments}

This work was supported by the Chinese National Natural Science Foundation under Contract nos. 51674106 and 51274091.

\section{References}

[1] C. S. Liu, C. P. Ren, and D. G. Li, "Reconstruction and deduction of cutting coal and rock load spectrum on modified discrete regularization algorithm," Journal of the China Coal Society, vol. 39, no. 5, pp. 981-986, 2014.

[2] C. S. Liu, C. P. Ren, and H. Fei, "Study on time-frequency spectrum characteristic of dynamic cutting load based on wavelet regularization," Applied Mechanics and Materials, vol. 577, pp. 196-200, 2014.

[3] Q. Tang, J. Dai, J. Liu, C. Liu, Y. Liu, and C. Ren, "Quantitative detection of defects based on Markov-PCA-BP algorithm using pulsed infrared thermography technology," Infrared Physics \& Technology, vol. 77, pp. 144-148, 2016.

[4] W. Mcilhagga, "The Canny edge detector revisited," International Journal of Computer Vision, vol. 91, no. 3, pp. 251-261, 2011.

[5] Q. J. Tang, J. M. Dai, C. S. Liu, Y. L. Liu, and C. P. Ren, "Study on defects edge detection in infrared thermal image based on ant colony algorithm," in Proceedings of the Ubiquitous Computing and Multimedia Applications 2016, vol. 137, pp. 37-41, Teresina, Brazil, November 2016.

[6] J. Y. Liu, Q. J. Tang, Y. Wang, Y. Lu, and Z. Zhang, 'Defects' geometric feature recognition based on infrared image edge detection," Infrared Physics \& Technology, vol. 67, pp. 387390, 2014.

[7] B. Mathieu, P. Melchior, A. Oustaloup, and C. Ceyral, "Fractional differentiation for edge detection," Signal Processing, vol. 83, no. 11, pp. 2421-2432, 2003.

[8] C. B. Gao, J. L. Zhou, J. R. Hu, and F. N. Lang, "Edge detection of colour image based on quaternion fractional differential," IET Image Processing, vol. 5, no. 3, pp. 261-272, 2011.

[9] J. Bai and X.-C. Feng, "Fractional-order anisotropic diffusion for image denoising," IEEE Transactions on Image Processing, vol. 16, no. 10, pp. 2492-2502, 2007.

[10] J. Józwik, "Identification and monitoring of noise sources of CNC machine tools by acoustic holography methods," Advances in Science and Technology Research Journal, vol. 10, no. 30, pp. 127-137, 2016.

[11] D. Chen, S. Sun, C. Zhang, Y. Chen, and D. Xue, "Fractionalorder TV-L2 model for image denoising," Central European Journal of Physics, vol. 11, no. 10, pp. 1414-1422, 2013.

[12] C. He, Y.-Q. Ye, B. Jiang, and X. Zhou, "A novel edge detection method based on fractional-order calculus mask," Acta Automatica Sinica, vol. 38, no. 5, pp. 776-787, 2012.

[13] D. Goddeke and R. Strzodka, "Cyclic reduction tridiagonal solvers on GPUs applied to mixed-precision multigrid," IEEE Transactions on Parallel \& Distributed Systems, vol. 22, no. 1, pp. 22-32, 2010.
[14] V. Parot, C. Sing-Long, C. Lizama, C. Tejos, S. Uribe, and P. Irarrazaval, "Application of the fractional fourier transform to image reconstruction in MRI," Magnetic Resonance in Medicine, vol. 68, no. 1, pp. 17-29, 2012.

[15] V. Melicher and V. Vrabel, "On a continuation approach in Tikhonov regularization and its application in piecewiseconstant parameter identification," Inverse Problems, vol. 29, no. 11, article 115008, 2013.

[16] T. Poggio, V. Torre, and C. Koch, "Computational vision and regularization theory," Nature, vol. 317, no. 6035, pp. 314-319, 1985.

[17] T. S. Jang, H. G. Sung, S. L. Han, and S. H. Kwon, "Inverse determination of the loading source of the infinite beam on elastic foundation," Journal of Mechanical Science and Technology, vol. 22, no. 12, pp. 2350-2356, 2008.

[18] J. M. Varah, "A practical examination of some numerical methods for linear discrete ill-posed problems," SIAM Review, vol. 21, no. 1, pp. 100-111, 1979.

[19] Y. Dai, L. Liu, and S. Feng, "On the identification of coupled pitch and heave motions using opposition-based particle swarm optimization," Mathematical Problems in Engineering, vol. 2014, Article ID 784049, 10 pages, 2014.

[20] W. Luo, "Parameter identifiability of ship manoeuvring modeling using system identification," Mathematical Problems in Engineering, vol. 2016, Article ID 8909170, 10 pages, 2016.

[21] C. P. Ren, N. J. Wang, and C. S. Liu, "Identification of random dynamic force using an improved maximum entropy regularization combined with a novel conjugate gradient," Mathematical Problems in Engineering, vol. 2017, Article ID 9125734, 14 pages, 2017.

[22] N. J. Wang, C. P. Ren, and C. S. Liu, "A novel fractional Tikhonov regularization coupled with an improved supermemory gradient method and application to dynamic force identification problems," Mathematical Problems in Engineering, vol. 2018, Article ID 4790950, 16 pages, 2018.

[23] C. S. Liu, "Novel algorithms based on the conjugate gradient method for inverting ill-conditioned matrices, and a new regularization method to solve ill-posed linear systems," Computer Modeling in Engineering and Sciences, vol. 60, pp. 279-308, 2010.

[24] C. S. Liu, "An optimally generalized steepest-descent algorithm for solving ill-posed linear systems," Journal of Applied Mathematics, vol. 2013, Article ID 154358, 15 pages, 2013.

[25] F. Baorong, Research on Fracture Detection Algorithm of Coal Rock Image, Jiangnan University, Wuxi, China, 2015.

[26] J. Xu, W. Wang, and L. Chen, "Image fusion algorithm for rock fracture detection using wavelet transform," in Proceedings of the 3rd International Symposium on Advanced Optical Manufacturing and Testing Technologies: Optical Test and Measurement Technology and Equipment, pp. 672337672339, Chengdu, China, November 2007.

[27] W. Weixing, W. Fengping, H. Xiaojun, and S. Junfang, "Rock fracture image acquisition using two kinds of lighting and fusion on a wavelet transform," Bulletin of Engineering Geology and the Environment, vol. 75, no. 1, pp. 311-324, 2016.

[28] Y. Zhang, Y. F. Pu, J. R. Hu, and J. L. Zhou, "A class of fractional-order variational image inpainting models," $A p$ plied Mathematics and Information Sciences, vol. 6, no. 26, pp. 299-306, 2012.

[29] P. Chen and H. Fang, "Existence of periodic and subharmonic solutions for second-order p-Laplacian difference equations," Advances in Difference Equations, vol. 2007, Article ID 042530, 10 pages, 2007. 
[30] J. Wei and C. Hui, "New edge detection model based on fractional differential and Sobel operator," Computer Engineering \& Applications, vol. 48, no. 4, pp. 182-185, 2012.

[31] X. Qing, H. Z. Qi, X. L. Jiang et al., “A modified edge extraction algorithm of infrared thermal image based on Sobel operator," Infrared Technology, vol. 37, pp. 462-466, 2015.

[32] R. Agarwal, "Modified bit-planes sobel operator: a new approach to edge detection," International Journal of Computer Applications, vol. 117, no. 7, pp. 9-15, 2015.

[33] R. El-Khazali, "On the biquadratic approximation of fractional-order Laplacian operators," Analog Integrated Circuits and Signal Processing, vol. 82, no. 3, pp. 503-517, 2015.

[34] G. Derfel, P. J. Grabner, and F. Vogl, "Laplace operators on fractals and related functional equations," Journal of Physics A Mathematical \& Theoretical, vol. 45, no. 46, article 463001, 2012.

[35] N. Otsu, "A threshold selection method from gray-level histograms," IEEE Transactions on Systems, Man, and Cybernetics, vol. 9, no. 1, pp. 62-66, 1979.

[36] R. L. Kirby and A. Rosenfeld, "A note on the use of (gray level, local average gray level) space as an aid in threshold selection," IEEE Transactions on Systems Man \& Cybernetics, vol. 9, pp. 860-864, 1979. 


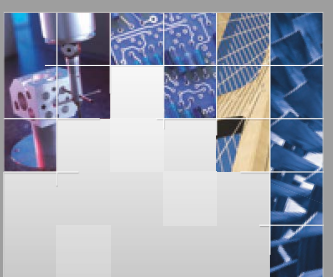

\section{Enfincering}
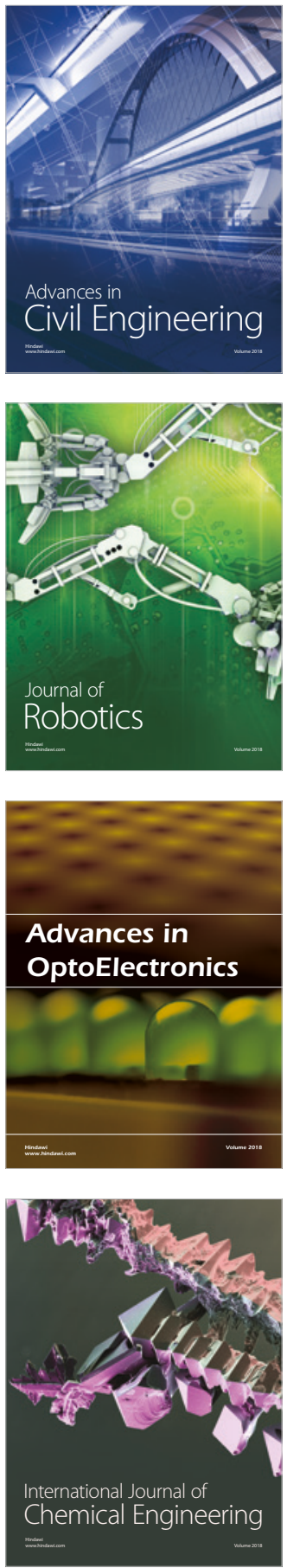

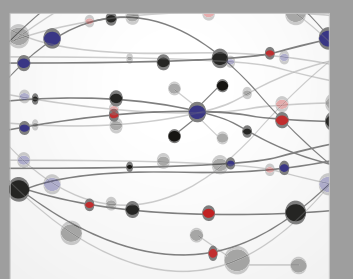

\section{Rotating \\ Machinery}

The Scientific World Journal

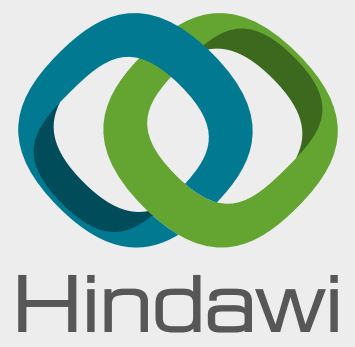

Submit your manuscripts at

www.hindawi.com
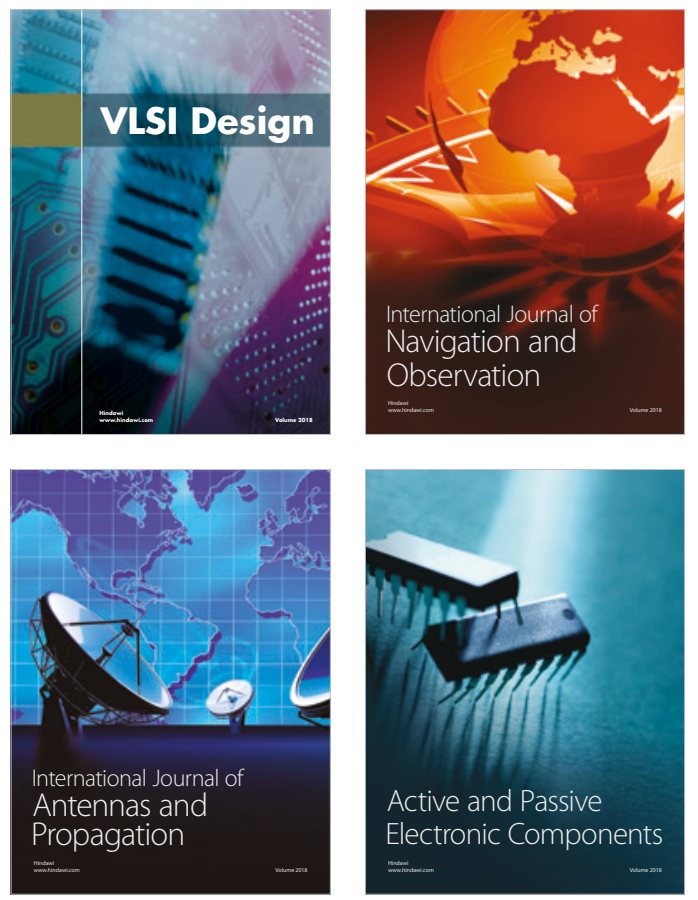
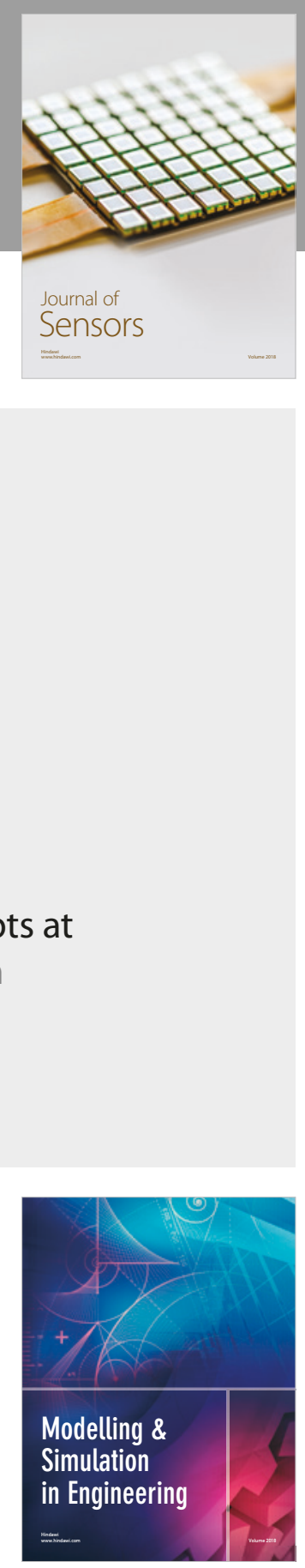

\section{Advances \\ Multimedia}
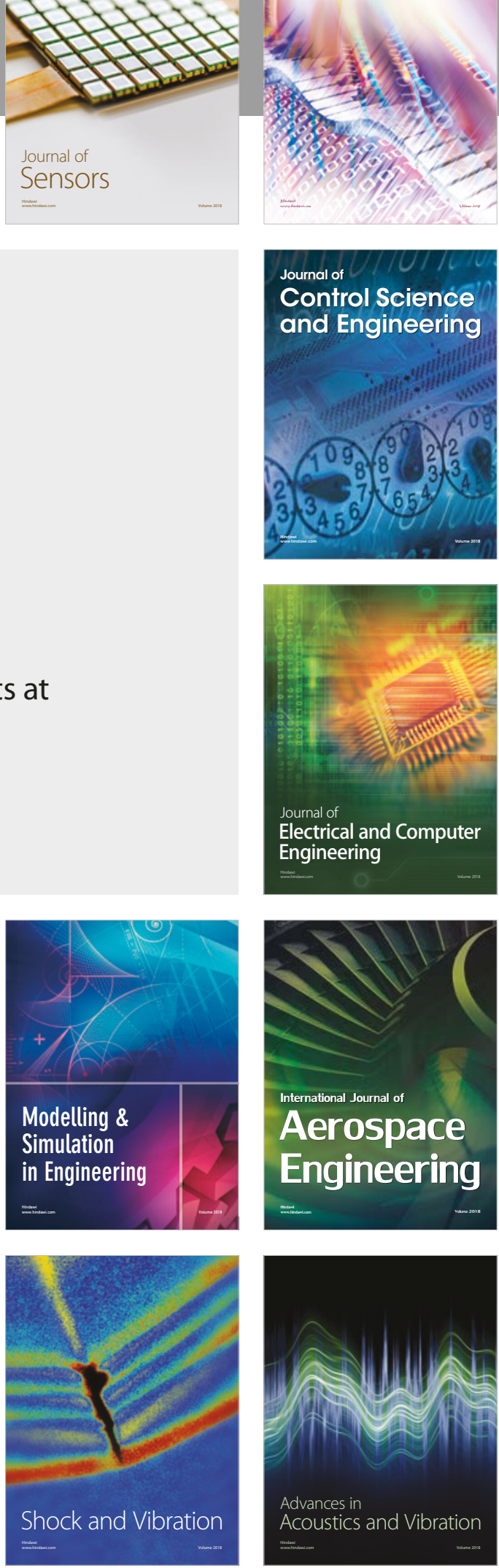